\title{
Clumpy Scattering Regions in Active Galactic Nuclei
}

\author{
Makoto Kishimoto \\ Department of Astronomy, Faculty of Science, Kyoto University, Kyoto \\ 606-01, Japan
}

\begin{abstract}
Simple unified models predict that many Seyfert 2s should be highly polarized ( $\gtrsim 50 \%$ ), but such highly polarized Seyfert 2 s are not observed. As one of the possibilities to explain this problem, the existence of clumpy scattering clouds with optically thick condensations in the nuclear regions is discussed.
\end{abstract}

\section{Introduction}

Unified models of AGNs have been supported by many observations. In particular, the recent imaging polarimetry of NGC 1068 has confirmed that the nuclear region actually consists of the point source of radiation and a scattering cloud. However, the simple unified model predicts that many Seyfert 2 s should be highly polarized ( $\gtrsim 50 \%$, Miller \& Goodrich 1990 ), which contradicts the observations. This has been one of the problems of the unified model. Two possibilities to explain this problem are discussed below.

\section{Low Polarization and Clumpy Scattering Clouds}

\subsection{Diluting Radiation}

It has been argued that certain unpolarized radiation from the scattering region itself is diluting the scattered (polarized) radiation. This seems to be true in some objects. Tran (1995) estimated the amount of this diluting radiation and found the intrinsic polarization degree of the scattered radiation to be around 10$30 \%$. This value, however, is still low compared to what is expected. Moreover, there is no evidence for diluting radiation in NGC 1068 (Antonucci et al. 1994; Tran 1995).

\subsection{Multiple Scattering}

If the scattering medium is optically thick, multiple scattering makes the polarization lower. However, in the unified model, the scattering region must be optically thin, otherwise broad lines would be seen in both of type 1 and type 2 objects. On the other hand, the HST image mentioned above revealed that the scattering cloud of NGC 1068 has a clumpy structure. A clumpy cloud can be optically thin, even if each clump is optically thick. The polarization of the scattered radiation from such a cloud is expected to be low, because of multiple scatterings in such a cloud. The radiation can easily enter deep inside the cloud 


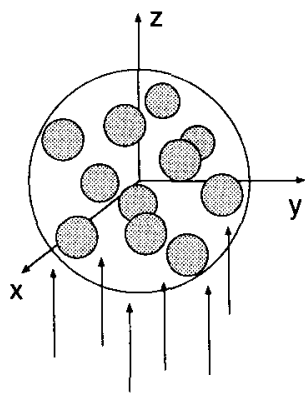

(a)

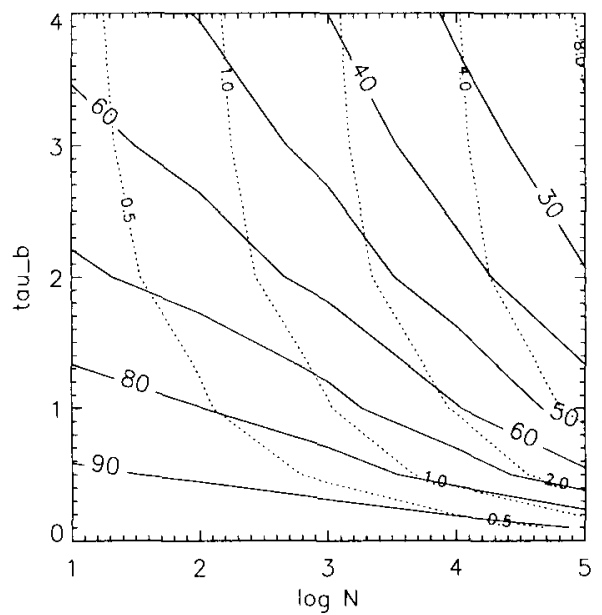

(b)

Figure 1. (a) Simple model of a clumpy cloud. Spherical clumps are randomly distributed in a spherical region. (b) The optical thickness for the diameter of the whole cloud is drawn in dotted lines for 0.5 , $1.0,2.0,4.0,8.0$. The solid lines indicate the polarization degree for an edge-on view.

and the photon which escaped from one blob can again go into another blob, thus the photon will often be multiply scattered.

We have calculated the polarization for a model of a clumpy cloud (Fig. 1a), taking into account the full effect of multiple scattering by a Monte-Carlo method. In Fig. 1b, we show the map of polarization and the cloud optical thickness for scattering, on the plane of $\left(N, \tau_{b}\right)$, where $N$ is the number of clumps in the cloud and $\tau_{b}$ is the scattering optical thickness of each clump in the cloud. We find that the polarization can be considerably low even if the whole clumpy cloud is optically thin, suggesting that the observed polarization could be much lower than that predicted by the simple unified model.

\section{Conclusions}

We are thus led to the possibility that clumpy clouds with optically thick clumps exist in AGNs, and this could be another cause of the low polarization. For more details, see Kishimoto (1996).

\section{References}

Antonucci, R., Hurt, T., \& Miller, J. S. 1994, ApJ, 430, 210.

Kishimoto, M. 1996, ApJ, 468, 606.

Miller, J. S., \& Goodrich, R. W. 1990, ApJ, 355, 456.

Tran, H. D. 1995, ApJ, 440, 565. 